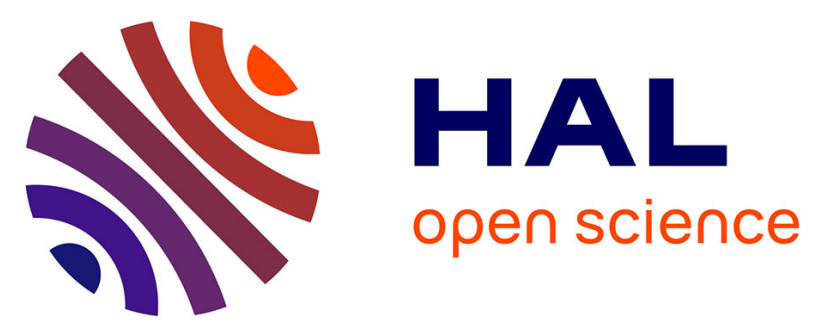

\title{
On-axis TKD for orientation mapping of nanocrystalline materials in SEM
}

Etienne Brodu, Emmanuel Bouzy, Jean-Jacques Fundenberger, Julien Guyon, Antoine Guitton, Yudong Zhang

\section{- To cite this version:}

Etienne Brodu, Emmanuel Bouzy, Jean-Jacques Fundenberger, Julien Guyon, Antoine Guitton, et al.. On-axis TKD for orientation mapping of nanocrystalline materials in SEM. Materials Characterization, 2017, 130, pp.92-96. 10.1016/j.matchar.2017.05.036 . hal-02392258

\section{HAL Id: hal-02392258 \\ https://hal.univ-lorraine.fr/hal-02392258}

Submitted on 6 Dec 2020

HAL is a multi-disciplinary open access archive for the deposit and dissemination of scientific research documents, whether they are published or not. The documents may come from teaching and research institutions in France or abroad, or from public or private research centers.
L'archive ouverte pluridisciplinaire HAL, est destinée au dépôt et à la diffusion de documents scientifiques de niveau recherche, publiés ou non, émanant des établissements d'enseignement et de recherche français ou étrangers, des laboratoires publics ou privés.

\section{(1) (1) $\$$}

Distributed under a Creative Commons Attribution - NonCommercial - NoDerivatives| 4.0 


\title{
On-axis TKD for orientation mapping of nanocrystalline materials in SEM
}

\author{
Etienne Brodu ${ }^{\mathrm{a}, \mathrm{b}}$, Emmanuel Bouzy ${ }^{\mathrm{a}, \mathrm{b}, *}$, Jean-Jacques Fundenberger ${ }^{\mathrm{a}, \mathrm{b}}$, Julien Guyon ${ }^{\mathrm{a}, \mathrm{b}}$, \\ Antoine Guitton ${ }^{\mathrm{a}, \mathrm{b}}$, Yudong Zhang ${ }^{\mathrm{a}, \mathrm{b}}$ \\ a Laboratoire d'Etude des Microstructures et de Mécanique des Matériaux (LEM3), UMR CNRS 7239, Université de Lorraine, 57045 Metz, France \\ b Laboratory of Excellence on Design of Alloy Metals for low-MAss Structures (DAMAS), University of Lorraine, 57045 Metz, France
}

\section{A R T I C L E I N F O}

\section{Keywords:}

Orientation mapping

Transmission Kikuchi Diffraction (TKD)

On-axis detector

Nanometer-sized grains

Lateral spatial resolution

\begin{abstract}
A B S T R A C T
A new configuration for Transmission Kikuchi Diffraction (TKD) in SEM was recently developed, with a scintillator perpendicular to the electron beam. This configuration, "on-axis" TKD, makes grains and twins below $10 \mathrm{~nm}$ well visible in orientation maps and with high indexation rate, as demonstrated with an electrodeposited nanocrystalline Ni. This high lateral spatial resolution is achieved by combining high accelerating voltage and low sample thickness to reduce the interaction volume. Among the advantages of the on-axis TKD, orientation mapping of particularly thin samples can be realized thanks to the advantageous axial position of the detector. Indeed, the reduction of the solid angle of the transmitted intensity around the beam axis with decreasing sample thickness is much less of a problem with an axial detector than with an off-axis detector. With an on-axis detector, the only condition for production of an orientation map is that the sample must be thick enough to produce Kikuchi diffraction in addition to spots.
\end{abstract}

\section{Introduction}

The analytical capabilities of Scanning Electron Microscopes (SEM) enlarge continuously. Among them, the diffraction acquisition capabilities offer the possibility for orientation mapping of microstructure constituents of materials. The Electron Backscatter Diffraction (EBSD) is currently the main achieved tool in this development. It is routinely and widely used to produce local orientation maps of a polished surface of bulk materials in the SEM, via the automated recording and analysis of Kikuchi diffraction patterns. Orientation maps can then be easily processed to extract the following information: phase distribution, grain size distribution, orientation of individual grains, disorientation inside grains and grain boundary characteristics [1]. Via advanced processing of orientation maps, one can access additional information. For example, from the analysis of the disorientation inside the grains, one can deduce the dislocation density referred to as GND (Geometrically Necessary Dislocations) [2,3], as well as the disclination density [4]. Also, a refined analysis of the Kikuchi diffraction patterns, typically by crosscorrelation, can allow improving the angular resolution of the technique and then even allow determining local strain gradients within the material $[5,6]$. EBSD is thus overall a very powerful technique of SEM.

One of the limits of EBSD though is its lateral resolution. Indeed, with a lateral resolution of about $50 \mathrm{~nm}$ [7-9], many features remain out of reach. With the objective of overcoming this limitation, a new diffraction detecting technique was recently developed in SEMs, referred to as TKD or t-EBSD [10]. TKD (Transmission Kikuchi Diffraction) technique uses electron transparent samples, prepared in the same way as for TEM, instead of polished-bulk samples as for EBSD. The Kikuchi diffraction patterns in TKD are then produced by the electrons going through the thin sample, instead of by the backscattered ones as for EBSD. In this way the electron-matter interaction volume of the sample for TKD is reduced with respect to that for EBSD, leading to an improvement in lateral resolution from about $50 \mathrm{~nm}$ for EBSD to under $10 \mathrm{~nm}$ for TKD [11-13]. Also, a major advantage of TKD is that it does not require hardware modification: one can use the EBSD hardware to produce TKD orientation maps. Indeed, the only difference lies in the preparation of the sample and its positioning in the chamber (see [10]).

In the recent development of TKD, an alternative configuration was developed at LEM3: "on-axis" TKD [14]. This technique involves a hardware modification in comparison to the conventional, off-axis TKD first proposed by Keller and Geiss [10]. The scintillator is moved from its vertical position in the conventional system to a horizontal position under the sample. The scintillator thus sits in the direction of the highest transmitted electron intensity, enabling much faster acquisition. It was previously estimated that on-axis TKD is 20 times faster than conventional TKD [15] (note that it depends on the angular distribution of the transmitted intensity and thus on sample thickness, incident energy and atomic number). With a much higher acquisition speed, on-

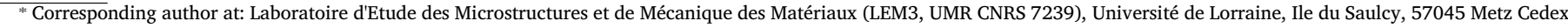
01, France.

E-mail address: emmanuel.bouzy@univ-lorraine.fr (E. Bouzy).
} 

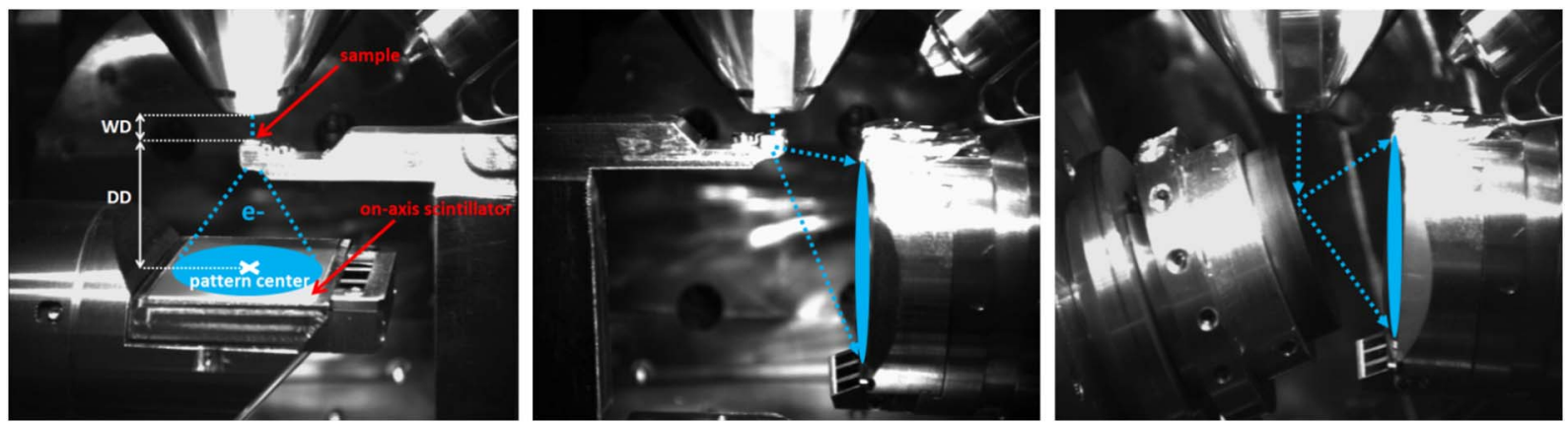

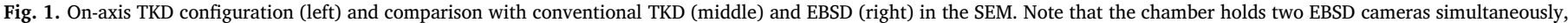
each on its own port, one for EBSD and conventional TKD, the other one being dedicated to on-axis TKD.
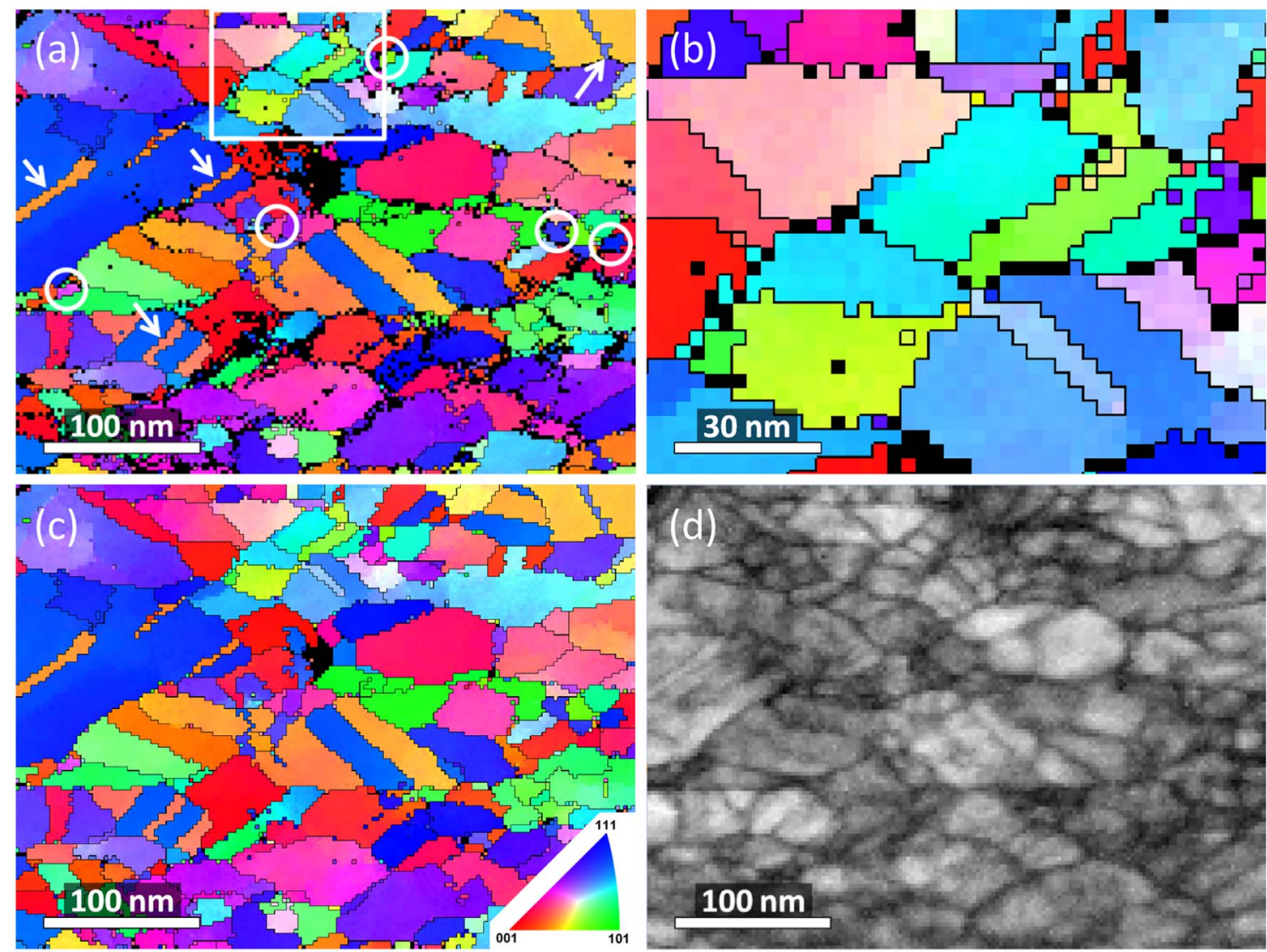

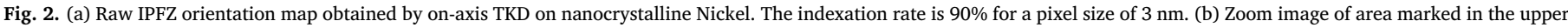

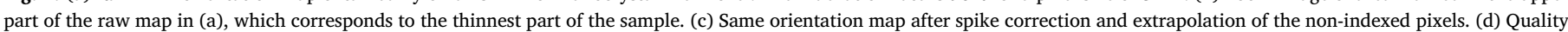
pattern map. All the measurement parameters are listed in Table 1 . White circles indicate grains with a size around $10 \mathrm{~nm}$ and white arrows indicate twins that are $6-12 \mathrm{~nm}$ wide.

axis TKD is more suitable than conventional TKD for the analysis of beam sensitive materials, as well as less affected by the lateral beam drift and the overall beam instabilities. The present study presents the first results of lateral resolution that the on-axis TKD system can achieve, using a sample with nanometer-sized grains. Some improvements with respect to the conventional TKD are also discussed.

\section{Experimental Set-up}

The orientation map on nanocrystalline nickel was obtained with a FEG-SEM Zeiss Supra 40 microscope equipped with a set-up for on-axis TKD (Transmission Kikuchi Diffraction) acquisition that consists of a Bruker e-Flash ${ }^{1000}$ camera with a Bruker OPTIMUS $^{\mathrm{TM}}$ detector. The specificity of this detector is that it positions the scintillator horizontally, instead of vertically in the conventional TKD system (Fig. 1). This on-axis detector was first designed, built and tested at LEM3 [14] and is now commercialized by Bruker under the name OPTIMUS ${ }^{\mathrm{TM}}$. With this on-axis configuration the sample is set perpendicular to the electron beam with the horizontal scintillator below it in order to collect the transmitted electrons through the sample. The diffraction patterns captured by the scintillator are then sent to the CCD camera, which remains vertical in the camera block, via a mirror inclined at $45^{\circ}$ to the direction of the transmitted electrons. The main advantage of this configuration is that the scintillator is positioned in the direction of the maximum signal intensity, enabling a very high acquisition speed, while the spatial resolution is expected to be at least as good as the conventional TKD. With the on-axis TKD, the distance between the detector and the sample is adjustable, typically in the range $5-30 \mathrm{~mm}$, which allows modulating the solid angle screened in the reciprocal space. This distance is adjusted by tilting the full camera block. The consequence is that the scintillator does not remain exactly perpendicular to the electron beam, although it remains in the range $90^{\circ} \pm 2^{\circ}$ 
degrees for a detector-sample distance in the range [5-30] $\mathrm{mm}$.

The material used for this study is nanocrystalline Ni. It was synthesized by electrodeposition in an electrolytic solution composed of nickel sulfate, nickel chloride additives and boric acid. This technique produces columnar grains along the growth direction. Additional information regarding this material can be found in [16]. Then, an electron transparent thin foil was prepared by Focused Ion Beam (FIB) milling according to the guidelines in [17], with the plane of the foil set perpendicular to the growth direction. The target thickness of $100-150 \mathrm{~nm}$ for the sample was reached, with the edges of the sample being much thinner. A final FIB milling at $5 \mathrm{keV}$; $50 \mathrm{pA}$, was performed in order to eliminate part of the amorphous layer induced during the FIB milling.

\section{Results and Discussion}

\subsection{Orientation Map on Nanocrystalline Nickel}

TKD produces orientation maps with much more details than EBSD thanks to an improvement of lateral spatial resolution. This improvement results from the reduction of electron-matter interaction volume in the sample. The interaction volume is indeed much smaller in transmission mode than in backscattering mode. This study presents the first results of lateral resolution that the on-axis configuration for TKD can achieve. Fig. 2 presents an orientation map obtained on the nanocrystalline $\mathrm{Ni}$, with a step size of $3 \mathrm{~nm}$. All the microscope and acquisition parameters are listed in Table 1. After a thorough testing, these parameters produced the best results for this particular sample. Note that the orientation map presented in Fig. $2 \mathrm{a}$ is raw (i.e. without postprocessing). Fig. 2c, on the other hand, presents the orientation map after spike correction and extrapolation of the pixels not indexed using the ATOM software [18]. In the present case, the map can safely be post-processed thanks to the high indexation rate: $90 \%$. The main result is that the grains as small as $10 \mathrm{~nm}$ are well resolved (see white circles in Fig. 2a), with very few pixels being not indexed between them. Twins of only 2-4 pixels wide are resolved as well, corresponding to 6-12 nm (see white arrows in Fig. 2a), which shows that on-axis TKD is a very well suited technique for the determination of the orientation of nanometric grains. This result demonstrates that the lateral resolution of the on-axis TKD falls in the same range as the conventional TKD. However, for the reasons described in the next section, the on-axis TKD may be superior to the conventional TKD in term of the lateral

Table 1

Microscope, acquisition and sample parameters associated to the orientation map in Fig. 2.

\begin{tabular}{ll}
\hline Microscope & Zeiss Supra 40 \\
\hline Configuration & On-axis Transmission Kikuchi Diffraction \\
Camera & Bruker e-Flash ${ }^{1000}$ camera \\
Detector & Bruker OPTIMUS ${ }^{\mathrm{TM}}$ detector head \\
Accelerating voltage & $30 \mathrm{keV}$ \\
Aperture & $60 \mu \mathrm{m}$ - high current mode (about 2.5 nA) \\
Working distance & $7.1 \mathrm{~mm}$ \\
Sample tilt & $0^{\circ}$ \\
Magnification & $243 \mathrm{~K} \times$ \\
Detector-sample distance & $19.7 \mathrm{~mm}$ \\
Acquisition/indexation software & Bruker Esprit 2.1 \\
Pattern resolution & $400 \times 400$ pixels \\
Hough resolution & $60 \mathrm{pixels}$ \\
Integration time & $15 \mathrm{~ms} \times 2$ images (30 ms total per pixel) \\
Acquisition time & 9 min $50 \mathrm{~s}$ \\
Map size & $160 \times 120$ pixels \\
Step size & $3 \mathrm{~nm}$ \\
Indexation rate & $90 \%$ \\
Min. bands for indexation & 5 bands \\
Material & Ni synthesized by pulsed electrodeposition \\
Sample thickness & Specified in text \\
\hline
\end{tabular}

resolution. The on-axis TKD is also fast, thanks to the detector set in the direction of the highest intensity of the transmitted electrons: it took only about $10 \mathrm{~min}$ to produce this orientation map $(160 \times 120$ pixels $)$, with good pattern quality $(400 \times 400$ pixels and image averaging $\times 2)$. A high acquisition speed here is crucial for the study of such small grains because the lateral beam drifts and beam instabilities become very destructive to some information in orientation map (continuity of grains and their morphology). This fast technique also allows obtaining several maps of different areas in a short time and thus build solid data from a sample (i.e. statistically relevant). See Ref. [15] for the study of the acquisition speed of the on-axis TKD.

\subsection{Choice of Microscope and Samples Parameters}

Discussing the lateral resolution associated to the map in Fig. 2 requires to differentiate the physical resolution from the effective resolution. The physical lateral resolution is given by the size of the electron-matter interaction volume, which can be estimated from the shortest distance between two non-correlated diffraction patterns, as proposed by Zaefferer [9]. The effective lateral resolution, however, corresponds to the resolution of the orientation map, as displayed, and can be evaluated from the lateral noise of grain boundaries, the size of the smallest objects detected, as well as from the number of pixels not indexed between grains. The effective resolution can end up differently (often better) from the physical resolution depending on the capacity of the software to figure out the predominant orientation from the acquired diffraction pattern generated by several orientations, which is typically the case in grain boundary areas. Clearly, the effective resolution still depends on the physical resolution and in order to improve the effective resolution, one must improve the physical resolution. The key factor lies then in the reduction of the electron-matter interaction volume, which can be realized by the "high accelerating voltage - low sample thickness" combination $[11,13]$. This can be easily proved by Monte Carlo simulation of the electron-matter interaction like CASINO [22]. The experimental parameters used to produce the orientation map in Fig. 2 were chosen precisely with the objective to limit the interaction volume (see Table 1). The accelerating voltage was set to $30 \mathrm{keV}$ (i.e. the highest voltage available). Also, the thinnest part of the sample was chosen for mapping, at the very edge of the sample. More precisely, the thickness of the thinnest part of the orientation map in Fig. 2 is estimated to be well below $100 \mathrm{~nm}$ according to the type of diffraction contrast (Fig. 3): a mix of bright Kikuchi bands and diffraction spots, and even a few patterns with almost only spots are typical of a very low sample thickness [19]. Note that the presence of the spots in addition to the Kikuchi diffraction is not detrimental to the band detection in the Hough transform during our experiments with the on-axis TKD. Other than the accelerating voltage and sample thickness, the choice of the aperture is also expected to affect the lateral resolution because it determines the probe size. For the sake of the present study, the probe size was determined on nanometric gold droplets at all apertures (from $20 \mu \mathrm{m}$ to $120 \mu \mathrm{m})$. With the aperture of $60 \mu \mathrm{m}$, the "high current mode", produces $2.5 \mathrm{nA}$ at $30 \mathrm{keV}$ and the probe size is about $8 \mathrm{~nm}$, whereas with the aperture of $30 \mu \mathrm{m}$ the "low current mode" produces $0.3 \mathrm{nA}$ at $30 \mathrm{keV}$ and the probe size is about $3 \mathrm{~nm}$. Surprisingly, the tests with the two apertures showed no obvious effect on the lateral resolution of orientation maps, although the probe size varied by a factor two. Consequently, the role of the probe size remains unclear so far. Deeper studies on the influence of the probe size on the lateral spatial resolution, especially for thin specimen, is our going work. For the present study, we took advantage of a reasonably high intensity $(2.5 \mathrm{nA}$ at $30 \mathrm{keV})$ with the aperture of $60 \mu \mathrm{m}$ at "high current mode". Extremely, the intensity could go up to $7.1 \mathrm{nA}$ at $120 \mu \mathrm{m}$ - "high current mode" with our current on-axis TKD set-up. 

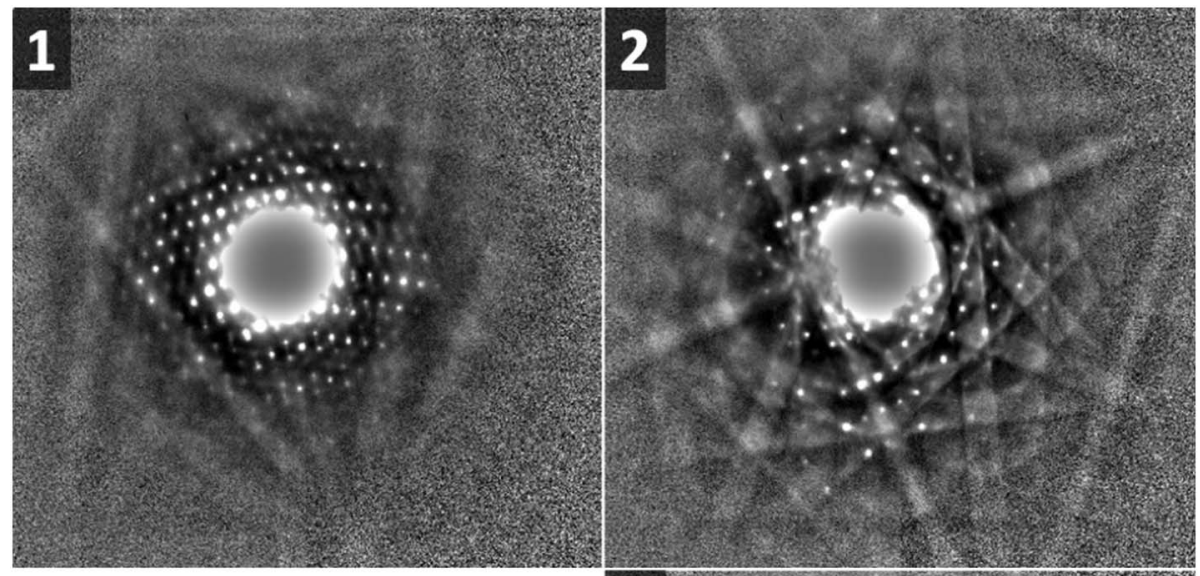

Fig. 3. Diffraction patterns associated to the orientation map in Fig. 2 for the estimation of the sample thickness. It is increasing from the top to the bottom of the map (i.e. the sample is thin at position 1 and thick at position 3, with position 2 being intermediate).
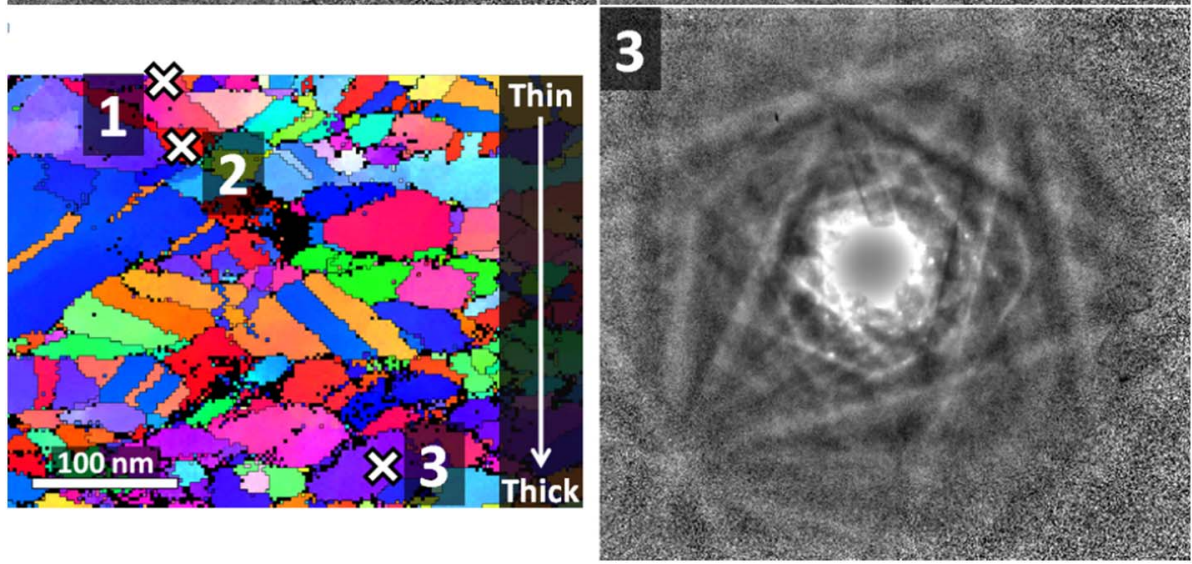

\subsection{Evaluation of Lateral Resolution}

Evaluating the physical and the effective resolutions associated to the orientation map in Fig. 2 is difficult for two reasons: 1- there is a thickness variation across the map, such that the sample is thinner in the upper-half of the map. It means that the resolution of the upper and lower part of the map would need to be discussed separately. 2- Because the TKD has a finite in-depth resolution $[20,21]$, the lateral resolution might vary depending on the angle between the grain boundary and the sample surface. Boundaries with an angle close to $90^{\circ}$ should produce less noise in the orientation maps, whereas boundaries with small angles could produce more noise. If we let aside point 2 , and focus on the upper part of the map, the effective resolution is in the range of 3-6 nm. Indeed, most grain boundaries present a lateral noise with amplitude within twice pixel size. In addition, grain boundaries present a remarkably low fraction of pixels that are not indexed. Fig. 2(b) displays a zoom image of the upper part of the map, allowing a presentation of the effective resolution. The physical resolution, however, is in the range of 6-9 $\mathrm{nm}$ for the present experiment again in the upper part of the map. It is evaluated from the distance between two un-convoluted diffraction patterns across grain boundaries. An example across a particular grain boundary is provided in Fig. 4, where we can see that the patterns number 2 and 3 contain traces of bands from two orientations whereas the patterns 1 and 4 are un-convoluted and present bands from single orientations.

We can see that the increase of the sample thickness from the top to the bottom of the map in Fig. 2 has a direct effect on the indexation rate. The lower-half of the map, from thicker sample part, corresponds a lower indexation rate, especially in grain boundary areas in comparison with the upper-half of the map, from thinner sample part. The reason is that a thicker sample produces bigger interaction volume, thus the diffraction patterns of the lower part of the map become blurred near the boundary areas by overlapping of several orientations. This emphasizes the need for thin samples if the goal is to achieve a high lateral resolution. By decreasing sample thickness, the interaction volume is reduced and thus the lateral resolution is improved. For this, the TKD with an on-axis detector is advantageous in terms of lateral resolution. Indeed it has been identified in the previous studies that very thin samples are challenging to study with the conventional TKD because the intensity reaching the detector is very low and the patterns contain consequently too little diffraction information for detection and indexation [11-13]. The reason is that while the interaction volume decreases with decreasing sample thickness, the transmitted intensity also tightens around the transmitted beam, resulting in very low intensity at high scattering angles, in particular, in the direction of the conventional TKD detector. This limitation is not present with the onaxis TKD because the scintillator sits right in the incident direction. The on-axis TKD can hence make use of thinner samples than the conventional TKD, which should give the on-axis TKD an advantage in term of lateral resolution (note that this advantage might be limited to the routine resolution and not be related to the ultimate resolution because the argument is "intensity based"). Besides, a limitation remains even for the on-axis TKD: the sample must be thick enough to produce Kikuchi diffraction, otherwise only diffraction spots are present (see Fig. 3). Also, right after the sample is thick enough to start producing Kikuchi diffraction, the bands and lines remain blurred for a short thickness interval before becoming sharp, as predicted by the kinematical theory of electron diffraction. It was previously observed with a silicon sample at $15 \mathrm{keV}$ that $20 \mathrm{~nm}$ of thickness was necessary for the lines and bands to reach a steady sharpness after their first generation [19].

\section{Conclusion}

A new diffraction technique, referred to as TKD or t-EBSD, was recently developed for SEM, with the objective to produce orientation 


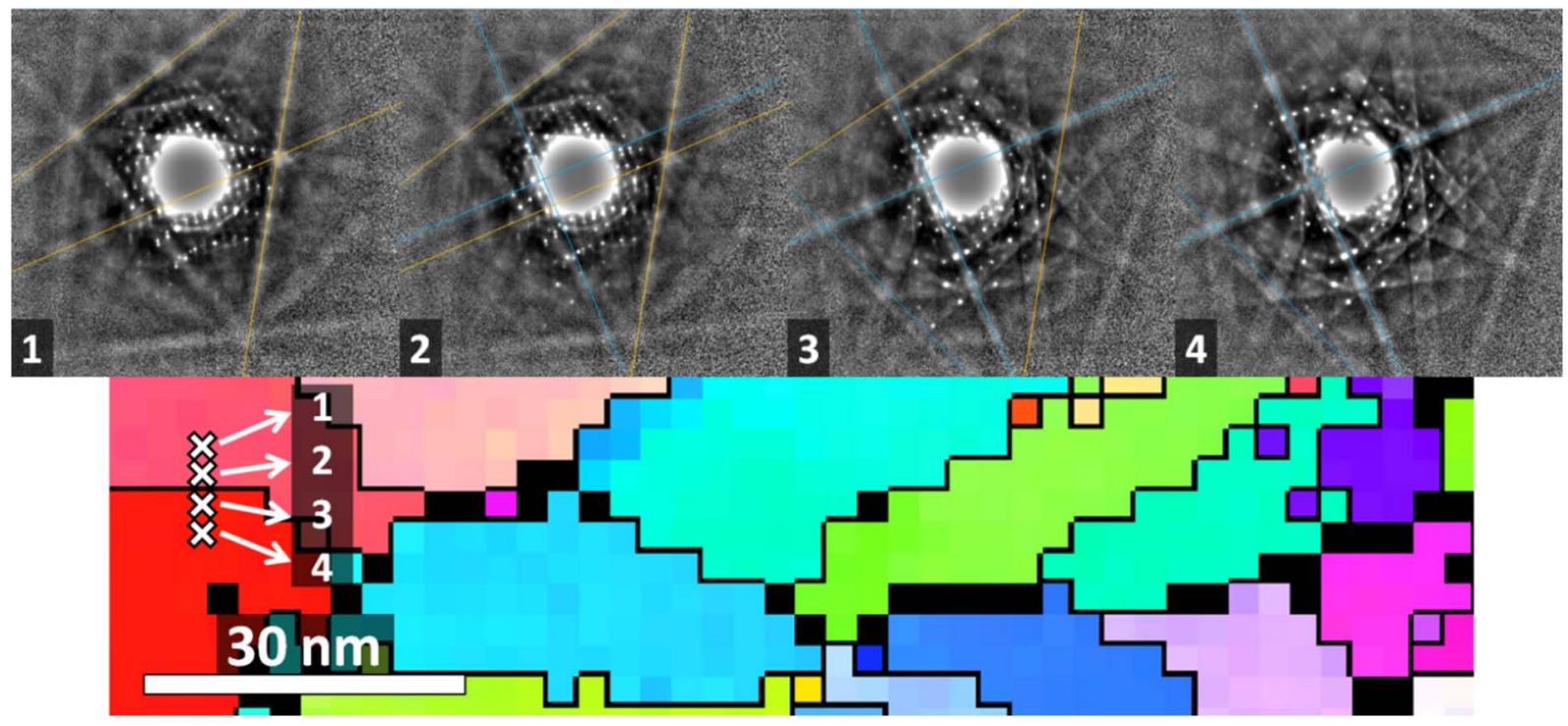

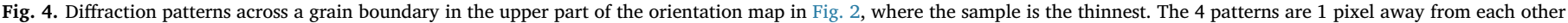
(pixel size $=3 \mathrm{~nm}$ ). All parameters are listed in Table 1 .

maps with a lateral spatial resolution largely improved in comparison with EBSD. As part of this development, a new configuration for TKD, the "on-axis TKD", was proposed, which involves a modification of the detector head: the scintillator is set horizontally, thus perpendicular to the electron beam, under the electron-transparent sample. This study shows that the lateral resolution provided by this new configuration falls in the same range as the conventional TKD, i.e. under $10 \mathrm{~nm}$. This makes it a suitable technique for the study of nanocrystalline materials. More precisely, an effective lateral resolution of 3-6 nm was achieved on a nanocrystalline nickel with the on-axis TKD, making grains and twins of $10 \mathrm{~nm}$ in size well visible and resolved, with a high indexation rate. It was achieved by using a combination of high accelerating voltage and a low sample thickness to reduce the electron-sample interaction volume. Besides, it is known that a small sample thickness can be challenging for the conventional TKD because the intensity reaching the detector becomes very low, which might induce a limit to the lateral resolution in routine experiments. It appeared that on-axis TKD does not suffer from such a limitation thanks to the axial position of the detector, the only condition is that the sample must be thick enough to produce Kikuchi diffraction, otherwise only spots are present on diffraction patterns. Future work still remains to be done to rigorously compare the ultimate lateral resolutions of conventional and on-axis TKD (even though they fall are in the same range anyway).

\section{Acknowledgements}

The authors are grateful to Karten DUST from the Technische Universität Darmstadt for providing nanocrystalline Ni samples. This work was supported by the French State through the program "Investment in the future" operated by the French National Research Agency (ANR) and referenced by ANR-11-LABX-0008-01 (LabEx DAMAS).

\section{References}

[1] R.A. Schwarzer, D.P. Field, B.L. Adams, M. Kumar, A.J. Schwartz, A.J. Schwartz, M. Kumar, B.L. Adams, D.P. Field (Eds.), Electron Backscatter Diffraction in Materials Science, vol. 2, Springer, New York, 2009.

[2] J.F. Nye, Some geometrical relations in dislocated crystals, Acta Metall. 1 (1953) 153-162.

[3] W. Pantleon, Resolving the geometrically necessary dislocation content by conventional electron backscattering diffraction, Scr. Mater. 58 (2008) 994-997.

[4] B. Beausir, C. Fressengeas, Disclination densities from EBSD orientation mapping, Int. J. Solids Struct. 50 (2013) 137-146.

[5] S. Villert, C. Maurice, C. Wyon, R. Fortunier, Accuracy assessment of elastic strain measurement by EBSD, J. Microsc. 233 (2009) 290-301.

[6] A. Wilkinson, Measurement of elastic strains and small lattice rotations using electron back scatter diffraction, Ultramicroscopy 62 (1996) 237-247.

[7] C.J. Harland, P. Akhter, J.A. Venables, Accurate microcrystallography at high spatial resolution using electron back-scattering patterns in a field emission gun scanning electron microscope, J. Phys. E Sci. Instrum. 14 (1981) 175-182.

[8] D.R. Steinmetz, S. Zaefferer, Towards ultrahigh resolution EBSD by low accelerating voltage, Mater. Sci. Technol. 26 (2010) 640-645.

[9] S. Zaefferer, On the formation mechanisms, spatial resolution and intensity of backscatter Kikuchi patterns, Ultramicroscopy 107 (2007) 245-266.

[10] R.R. Keller, R.H. Geiss, Transmission EBSD from $10 \mathrm{~nm}$ domains in a scanning electron microscope, J. Microsc. 245 (2012) 245-251.

[11] R. van Bremen, D. Ribas Gomes, L.T.H. de Jeer, V. Ocelík, J.Th.M. De Hosson, On the optimum resolution of transmission-electron backscattered diffraction (t-EBSD), Ultramicroscopy 160 (2016) 256-264.

[12] W. Trimby, Orientation mapping of nanostructured materials using transmission Kikuchi diffraction in the scanning electron microscope, Ultramicroscopy 12 (2012) $16-24$.

[13] Y.Z. Wang, M.G. Kong, Z.W. Liu, C.C. Lin, Y. Zeng, Effect of microscope parameter and specimen thickness of spatial resolution of transmission electron backscatter diffraction, J. Microsc. 264 (2016) 34-40.

[14] J.J. Fundenberger, E. Bouzy, D. Goran, J. Guyon, H. Yuan, A. Morawiec, Orientation mapping by transmission-SEM with an on-axis detector, Ultramicroscopy 161 (2016) 17-22.

[15] H. Yuan, E. Brodu, C. Chen, E. Bouzy, J.-J. Fundenberger, L.S. Toth, On-Axis versus off-Axis Transmission Kikuchi Diffraction technique: application to the characterization of severe plastic deformation induced ultrafine grained microstructures, J. Microsc. 0 (2017) 1-11.

[16] Z. Sun, S. Van Petegem, A. Cervellino, K. Durst, W. Blum, H. Van Swygenhoven, Dynamic recovery in nanocrystalline Ni, Acta Mater. 91 (2015) 91-100.

[17] M. Schaffer, B. Schaffer, Q. Ramasse, Sample preparation for atomic-resolution STEM at low voltages by FIB, Ultramicroscopy 114 (2012) 62-71.

[18] B. Beausir, J.-J. Fundenberger, Université de Lorraine - Metz, 2015, ATOM-Analysis Tools for Orientation Maps. http://atom-software.eu/.

[19] E. Brodu, E. Bouzy, J.-J. Fundenberger, Diffraction contrast dependence on sample thickness and incident energy in on-axis Transmission Kikuchi Diffraction in SEM, Ultramicroscopy 181 (2017) 123-133.

[20] K.P. Rice, R.R. Keller, M.P. Stoykovich, Specimen-thickness effects on transmission Kikuchi patterns in the scanning electron microscope, J. Microsc. 254 (2014) 129-136.

[21] P.W. Trimby, Y. Cao, Z. Chen, S. Han, K.J. Hemker, J. Lian, X. Liao, P. Rottmann, S. Samudrala, J. Sun, J.T. Wang, J. Wheeler, J.M. Cairney, Characterizing deformed ultrafine-grained and nanocrystalline materials using transmission Kikuchi diffraction in a scanning electron microscope, Acta Mater. 62 (2014) 69-80.

[22] D. Drouin, A.R. Couture, D. Joly, X. Tastet, V. Aimez, R. Gauvin, CASINO V2.42 - A fast and easy-to-use modeling tool for scanning electron microscopy and microanalysis users, SCANNING 29 (2007) 92-101. 